\title{
Oligoarticular vs Polyarticular Psoriatic Arthritis: A Longitudinal Study Showing Similar Characteristics
}

\author{
Dafna D. Gladman ${ }^{1}\left(\mathbb{D}\right.$, Justine Y. Ye², Vinod Chandran ${ }^{3}$, Ker-Ai Lee ${ }^{4}$, and Richard J. Cook ${ }^{4}$
}

ABSTRACT. Objective. The objectives of this study were to determine whether patients with oligoarticular presentation differ from those with polyarticular presentation and to identify potential predictors for evolution of oligoarthritis to polyarthritis in patients with psoriatic arthritis (PsA).

Methods. Patients who entered the University of Toronto PsA clinic between 1978 and 2018 within 12 months of diagnosis were identified. Only patients with $\geq 2$ clinic visits were included. Patients were followed at 6- to 12-month intervals according to standard protocol, which included demographics, clinical history, detailed clinical examination, laboratory information, and patient questionnaires. Radiographs were done at 2-year intervals. Oligoarthritis was defined by the presence of $\leq 4$ inflamed joints and progression as an increase to $\geq 5$ joints. Statistical analyses included logistic regression models as well as Weibull regression models, adjusted for age, disease duration, and sex.

Results. Of 407 patients, 192 (47\%) presented with oligoarthritis. Whereas demographic features were similar to those with polyarthritis, more patients with polyarthritis presented with dactylitis and enthesitis. Similar joint distribution was observed, with small joints of the hands and feet being most commonly affected. Patients with polyarthritis had higher Health Assessment Questionnaire and lower 36-item Short Form Health Survey (SF-36) scores. Of the 192 oligoarticular patients, 117 (61\%) remained oligoarticular and 75 (39\%) progressed to polyarthritis. A lower SF-36 mental component summary (MCS) score was the predictor for progressing to polyarthritis.

Conclusion. Oligoarticular PsA occurs in $47 \%$ of patients with PsA and is similar to polyarticular disease, with most patients having small joint involvement. The only predictor for progression to polyarthritis was lower SF-36 MCS.

Key Indexing Terms: oligoarthritis, outcome, polyarthritis, progression, psoriatic arthritis

The University of Toronto Psoriatic Arthritis Program is supported by a grant from the Krembil Foundation. VC was supported by a Pfizer Chair Rheumatology Research Award from the Department of Medicine, University of Toronto.

${ }^{I}$ D.D. Gladman, MD, FRCPC, Schroeder Arthritis Institute, Krembil Research Institute, University Health Network, Institute of Medical Science, University of Toronto, and Psoriatic Arthritis Program, University Health Network, Centre for Prognosis Studies in The Rheumatic Diseases, Toronto Western Hospital, Toronto; ${ }^{2}$ J.Y. Ye, MS, Psoriatic Arthritis Program, University Health Network, Centre for Prognosis Studies in The Rheumatic Diseases, Toronto Western Hospital, Toronto ${ }^{3} \mathrm{~V}$. Chandran, MBBS, MD, $D M, P h D$, Schroeder Arthritis Institute, Krembil Research Institute, University Health Network, Institute of Medical Science, University of Toronto, Psoriatic Arthritis Program, University Health Network, Centre for Prognosis Studies in The Rheumatic Diseases, Toronto Western Hospital, and Department of Laboratory Medicine and Pathobiology, University of Toronto, Toronto; ${ }^{4}$ K.A. Lee, MMath, R.J. Cook, PhD, Department of Statistics and Actuarial Science, University of Waterloo, Waterloo, Ontario, Canada.

The authors declare no conflict of interest relevant to this article.

Address correspondence to Dr. D.D. Gladman, Director, Psoriatic Arthritis

Program, Centre for Prognosis Studies in the Rheumatic Diseases,

University Health Network, Toronto Western Hospital, Schroeder Arthritis

Institute, 399 Bathurst St., Toronto, ON M5T 2S8, Canada.

Email:dafna.gladman@utoronto.ca.

Accepted for publication July 14, 2021.
In Moll and Wright's original description of psoriatic arthritis (PsA), the authors identified oligoarticular disease, defined as $\leq 4$ joints, as the most frequent pattern observed. ${ }^{1}$ However, the prevalence of oligoarticular disease has varied in subsequently reported series. ${ }^{2,3}$ Moreover, it has been demonstrated that the patterns do not necessarily remain stable over time. ${ }^{4}$ Patients may present with oligoarticular disease but become polyarticular over time. Others may start with polyarticular disease but may reduce their joint counts either due to treatment or by the natural course of disease. Helliwell, et al suggested that patients with PsA should be classified as having peripheral arthritis, axial disease, or both. ${ }^{5}$ The Group for Research and Assessment of Psoriasis and Psoriatic Arthritis (GRAPPA) proposed that PsA should be considered as having domains rather than patterns. ${ }^{6}$ These domains include peripheral arthritis (which may include distal, oligoarticular, and polyarticular), axial disease, dactylitis, enthesitis, and skin and nail involvement.

In the past 2 decades, many new medications have been approved for the treatment of PsA.,8 Many of the randomized controlled trials required 5 tender and swollen joints, whereas others required only 3 tender and swollen joints and had an average of 20 tender and 15 swollen joints; in some jurisdictions, patients with oligoarticular disease $(<5$ joints) are not able to 
receive biologic therapy. ${ }^{9}$ It remains unclear whether patients with oligoarticular disease differ from those with polyarticular disease and what characteristics predict progression from oligoarticular to polyarticular disease.

The objectives of this study were to (1) determine whether patients with oligoarticular presentation differ from those with polyarticular presentation in terms of demographics, clinical characteristics, and treatment; and (2) identify potential predictors for the evolution of oligoarthritis to polyarthritis in patients with PsA.

\section{METHODS}

Setting. The study was conducted at the University of Toronto PsA Program where patients have been followed prospectively since 1978. This study was approved by the University Health Network Research Ethics Board (REB No. 08-0630-AE); patients consented to this study and agreed to the publication of the material.

Patient selection. Patients who entered the clinic within 12 months of diagnosis between 1978 and 2018 were identified. Only patients with at least 2 clinic visits were included.

Patient assessments. Patients were followed according to a standard protocol at 6- to 12-month intervals. The following were collected at each scheduled clinic visit: demographics, clinical history, and detailed clinical examination including 68 tender and 66 swollen joint counts (the collected information has been consistent since the initiation of the clinic); and laboratory information. Radiographs were performed at 2-year intervals. These patients had completed patient-reported outcomes annually, including the Health Assessment Questionnaire (HAQ) and the 36-item Short Form Health Survey (SF-36). The information was recorded in a computerized database. ${ }^{10,11}$ Physicians involved in patient care were instructed on the procedure for patient assessments; these clinic assesssments were therefore carried out uniformly. These assessments have been demonstrated to be reliable. ${ }^{12,13}$

Definition of outcomes. Two definitions of oligoarthritis were used. First, oligoarthritis was defined based on the presence of $\leq 4$ inflamed (tender and/or swollen) joints (out of a total of 68 tender and/or 66 swollen joints recorded for each patient). Tender and/or swollen joints are considered inflamed. We have demonstrated that patients with PsA, unlike with rheumatoid arthritis, may have inflammatory changes on magnetic resonance imaging even if the joint is just tender and not swollen. ${ }^{14}$ Since patients with PsA may have had inflamed joints prior to entry at the clinic but may have developed damage as a consequence of previous inflammation, ${ }^{15,16}$ we also used a definition of $\leq 4$ involved joints (including both inflamed and damaged joints).

Polyarthritis was defined as $\geq 5$ inflamed joints in the first instance, or $\geq 5$ involved joints in the second, as defined above. Progression was defined by the transition from oligoarthritis to polyarthritis, which is known to have occurred upon the first visit the joint count exceeded 4.

Statistical analysis. Descriptive statistics were computed to describe patients presenting with oligoarthritis or polyarthritis upon recruitment. Logistic regression models were fitted for the binary response indicating presentation with polyarthritis vs oligoarthritis. ORs, $95 \% \mathrm{CIs}$, and $P$ values were reported for each covariate. For individuals presenting with oligoarthritis, Weibull regression models were fitted to model the time of progression to polyarticular disease, accommodating left truncation and interval censoring of the progression time. ${ }^{17} \mathrm{HRs}, 95 \% \mathrm{CIs}$, and $P$ values were reported from these fitted models which adjusted for age, duration of PsA, and sex; analysis was restricted to patients who had complete covariate information. All significance thresholds were set at $P<0.05$. Descriptive statistics were computed, logistic regression models were fitted using SAS 9.4 (SAS Institute), and Weibull regression models fitted using TIBCO SpotFire S+ Version 8.2.0 (TIBCO Software Inc.).

\section{RESULTS}

There were 407 patients included in the analyses. Of those, 192 (47\%) presented with oligoarthritis and 215 (53\%) with polyarthritis. Demographic features were similar between the 2 groups (Table 1). As expected, polyarticular involvement was associated with a higher number of actively inflamed joints. More patients who presented with polyarticular disease had dactylitis and enthesitis. However, the distribution of joints involved was similar in the 2 groups, with small joints of the hands and feet being most commonly affected (Table 1). Patients with polyarticular presentation had higher HAQ scores, and lower SF-36 physical (PCS) and mental component summary (MCS) scores, reflecting reduced function.

Table 2 presents the results of a logistic regression analysis with polyarthritis vs oligoarthritis as outcome for all 407 patients but includes only those variables available for all patients at baseline. Involvement of the lower extremity small joints had the highest ORs for presentation with polyarthritis, but it should be noted that the CIs are wide.

Of the 407 patients, 228 had complete baseline covariate information. The results from fitting a logistic regression model with polyarthritis vs oligoarthritis as outcome is presented in Table 3. Again, involvement of the lower extremity small joints (in the full multivariate model) had the highest ORs for presentation with polyarthritis, albeit with wide CIs. In this analysis, which included patient reported outcomes, a lower SF-36 PCS was associated with polyarticular presentation. There were no differences in the presence of dactylitis, enthesitis, axial disease, Psoriasis Area Severity Index score, elevated acute-phase reactant, or medication use.

Of the 192 patients who presented with oligoarthritis, $117(61 \%)$ remained oligoarticular and 75 (39\%) evolved into polyarticular disease. We developed 2 Weibull regression models to examine the progression from oligoarthritis to polyarthritis. All models were adjusted for age, disease duration, and sex, and included only patients who had complete covariate information at each clinic visit. The first analysis that excluded dactylitis, enthesitis, and axial disease included 136 oligoarticular patients, of whom 46 evolved into polyarthritis. This model revealed that the presence of upper extremity small joints predicted progression, whereas higher SF-36 PCS (better function) protected from progression to polyarthritis (Table 4). The second Weibull regression model included dactylitis, enthesitis, and axial disease, and demonstrated that only lower SF-36 MCS was significantly associated with progression to polyarthritis (Table 5). It should be noted that treatment did not have an effect on progression in either model.

When we considered the definition of oligoarthritis based on involved (inflamed and/or damaged) joints, the number of patients with oligoarthritis reduced to 185 , with $39.5 \%$ having evolved into polyarticular disease, as some patients had evidence of damage that increased the number of involved joints. However, the demographic and clinical features are similar (Table 1). The factors associated with progression remained the same (results not shown). 


\begin{tabular}{|c|c|c|c|c|}
\hline & \multicolumn{2}{|c|}{ Based on Active ${ }^{\mathrm{a}}$ Joints } & \multicolumn{2}{|c|}{ Based on Involved ${ }^{\mathrm{b}}$ Joints } \\
\hline & Oligoarthritis & Polyarthritis & Oligoarthritis & Polyarthritis \\
\hline No. of patients, $\mathrm{n}$ & 192 & 215 & 185 & 222 \\
\hline Males, n (\%) & $117(60.9)$ & $121(56.3)$ & $112(60.5)$ & $126(56.8)$ \\
\hline Age at psoriasis diagnosis, yrs & $29.1(14.6)$ & $31.4(16.1)$ & $28.6(14.4)$ & $31.7(16.0)$ \\
\hline Age at PsA diagnosis, yrs & $42.5(12.9)$ & $43.6(13.3)$ & $42.0(12.7)$ & $43.9(13.4)$ \\
\hline BMI & $28.5(6.4)$ & $30.5(7.8)$ & $28.3(5.9)$ & $30.7(8.1)$ \\
\hline PASI & $4.0(7.1)$ & $5.1(9.3)$ & $4.1(7.1)$ & $5.1(9.2)$ \\
\hline Actively inflamed joints & $1.8(1.4)$ & $13.8(9.5)$ & $1.8(1.4)$ & $13.5(9.6)$ \\
\hline Dactylitis, \% & 16.1 & 34.9 & 15.1 & 35.1 \\
\hline Enthesitis, \% & 16.1 & 28.8 & 16.2 & 28.4 \\
\hline Axial disease, $\%$ & 11.5 & 17.2 & 10.8 & 17.6 \\
\hline LE LJ & 21.4 & 50.7 & 20.0 & 51.8 \\
\hline HAQ-DI & $0.4(0.4)$ & $0.7(0.6)$ & $0.4(0.4)$ & $0.7(0.6)$ \\
\hline SF-36 PCS & $43.7(10.5)$ & $37.0(11.3)$ & $43.7(10.5)$ & $37.1(11.3)$ \\
\hline SF-36 MCS & $45.5(11.0)$ & $43.7(10.5)$ & $45.6(11.0)$ & $43.6(10.5)$ \\
\hline \multicolumn{5}{|l|}{ Highest medication, \% } \\
\hline NSAIDs & 34.9 & 28.8 & 35.7 & 28.4 \\
\hline DMARDs & 13.5 & 17.7 & 13.0 & 18.0 \\
\hline Biologics & 5.7 & 3.7 & 5.9 & 3.6 \\
\hline
\end{tabular}

Values are expressed as mean (SD) unless otherwise indicated. ${ }^{a}$ Active joints: tender and/or swollen joints. ${ }^{\text {b }}$ Involved joints: active and/or damaged joints. DMARD: disease-modifying antirheumatic drug; HAQ-DI: Health Assessment Questionnaire-Disability Index; LE: lower extremity; LJ: large joint; MCS: mental component summary score; NSAID: nonsteroidal antiinflammatory drugs; PASI: Psoriasis Area Severity Index; PCS: physical component summary score; PsA: psoriatic arthritis; SF-36: 36-item Short Form Health Survey; SJ: small joint; UE: upper extremity.

Table 2. Features associated with presenting with polyarthritis vs oligoarthritis based on all patients ${ }^{\mathrm{a}}$.

\begin{tabular}{|c|c|c|c|c|c|c|}
\hline \multirow[t]{3}{*}{ Covariate } & \multicolumn{2}{|c|}{ Univariate Analysis } & \multicolumn{4}{|c|}{ Multivariate Analysis } \\
\hline & \multirow[b]{2}{*}{ OR $(95 \% \mathrm{CI})$} & \multirow[b]{2}{*}{$P$} & \multicolumn{2}{|c|}{ Full Model } & \multicolumn{2}{|c|}{ Reduced Model } \\
\hline & & & OR (95\% CI) & $P$ & OR (95\% CI) & $P$ \\
\hline PsA duration, yrs & & & $1.42(0.88-2.32)$ & 0.15 & $1.41(0.89-2.21)$ & 0.14 \\
\hline $\operatorname{Sex}(\mathrm{M}$ vs F) & & & $0.81(0.44-1.51)$ & 0.53 & $0.93(0.51-1.69)$ & 0.80 \\
\hline LE SJ & $13.52(8.30-22.03)$ & $<0.001$ & $17.80(9.17-34.55)$ & $<0.001$ & $17.15(9.04-32.56)$ & $<0.001$ \\
\hline UE LJ & $5.03(3.20-7.90)$ & $<0.001$ & $5.14(2.62-10.08)$ & $<0.001$ & $4.71(2.45-9.03)$ & $<0.001$ \\
\hline Enthesitis & $2.12(1.30-3.46)$ & 0.003 & $1.62(0.76-3.45)$ & 0.21 & & \\
\hline PASI & $1.02(0.99-1.04)$ & 0.18 & $1.03(0.99-1.07)$ & 0.19 & & \\
\hline \multicolumn{7}{|l|}{ Highest medication } \\
\hline NSAIDs & $0.74(0.47-1.16)$ & 0.19 & $0.62(0.31-1.20)$ & 0.16 & & \\
\hline
\end{tabular}

${ }^{a}$ Based on 407 patients with only the covariate information available at baseline clinic visit (215 with polyarthritis, 192 with oligoarthritis). DMARD: disease-modifying antirheumatic drug; LE: lower extremity; LJ: large joint; NSAID: nonsteroidal antiinflammatory drugs; PASI: Psoriasis Area Severity Index; PsA: psoriatic arthritis; SJ: small joint; UE: upper extremity. 
Table 3. Features associated with presenting with polyarthritis vs oligoarthritis based on 228 patients $^{\text {. }}$.

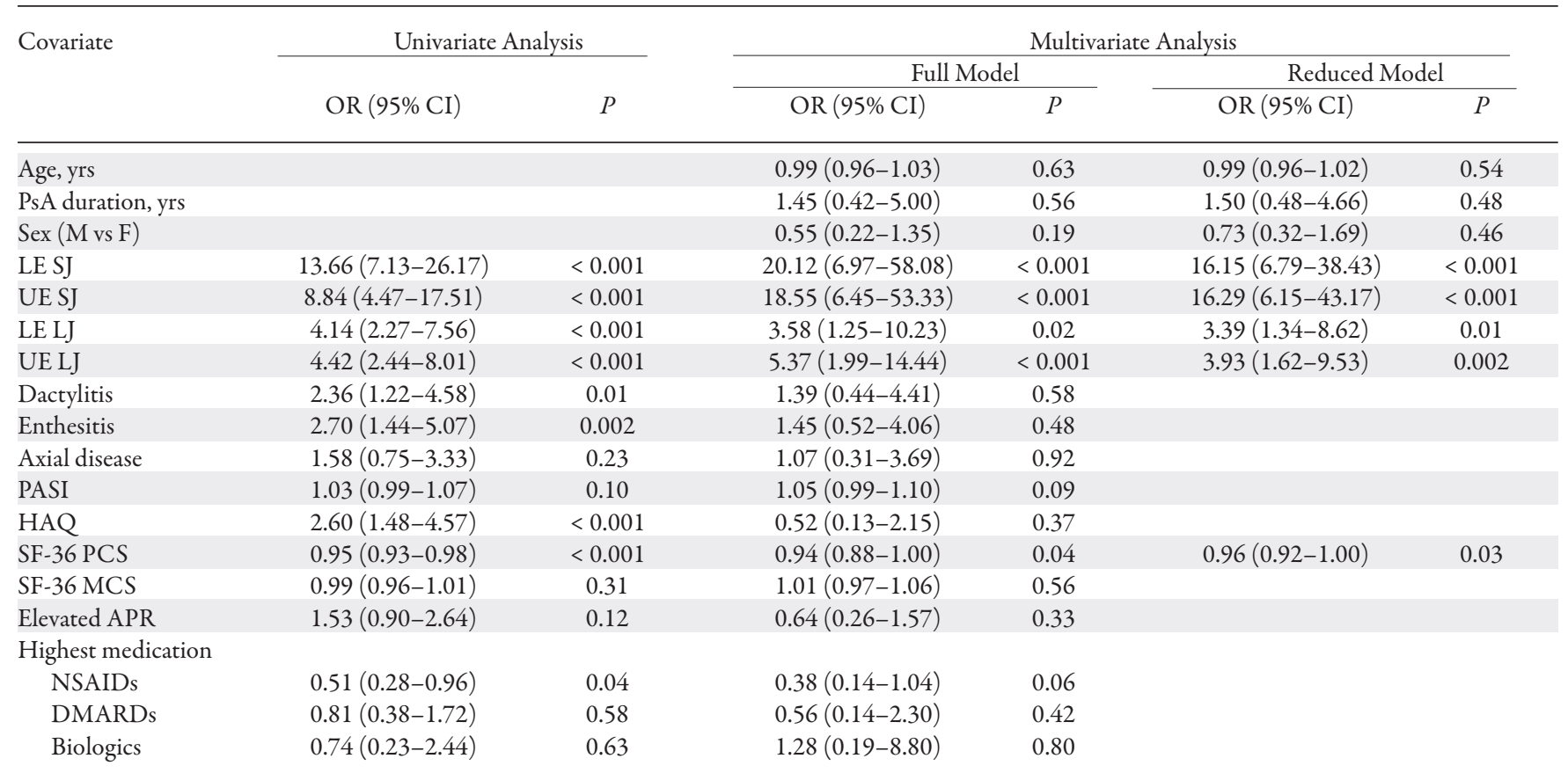

${ }^{a}$ Based on 228 patients with complete covariate information at baseline clinic visit (126 with polyarthritis, 102 with oligoarthritis). APR: acute-phase reactant; DMARD: disease-modifying antirheumatic drug; HAQ-DI: Health Assessment Questionnaire-Disability Index; LE: lower extremity; LJ: large joint; MCS: mental component summary score; NSAID: nonsteroidal antiinflammatory drugs; PASI: Psoriasis Area Severity Index; PCS: physical component summary score; PsA: psoriatic arthritis; SF-36: 36-item Short Form Health Survey; SJ: small joint; UE: upper extremity.

Table 4. Features associated with progression from oligoarthritis to polyarthritis based on 136 patients (excluding dactylitis, enthesitis, and axial disease) ${ }^{a}$.

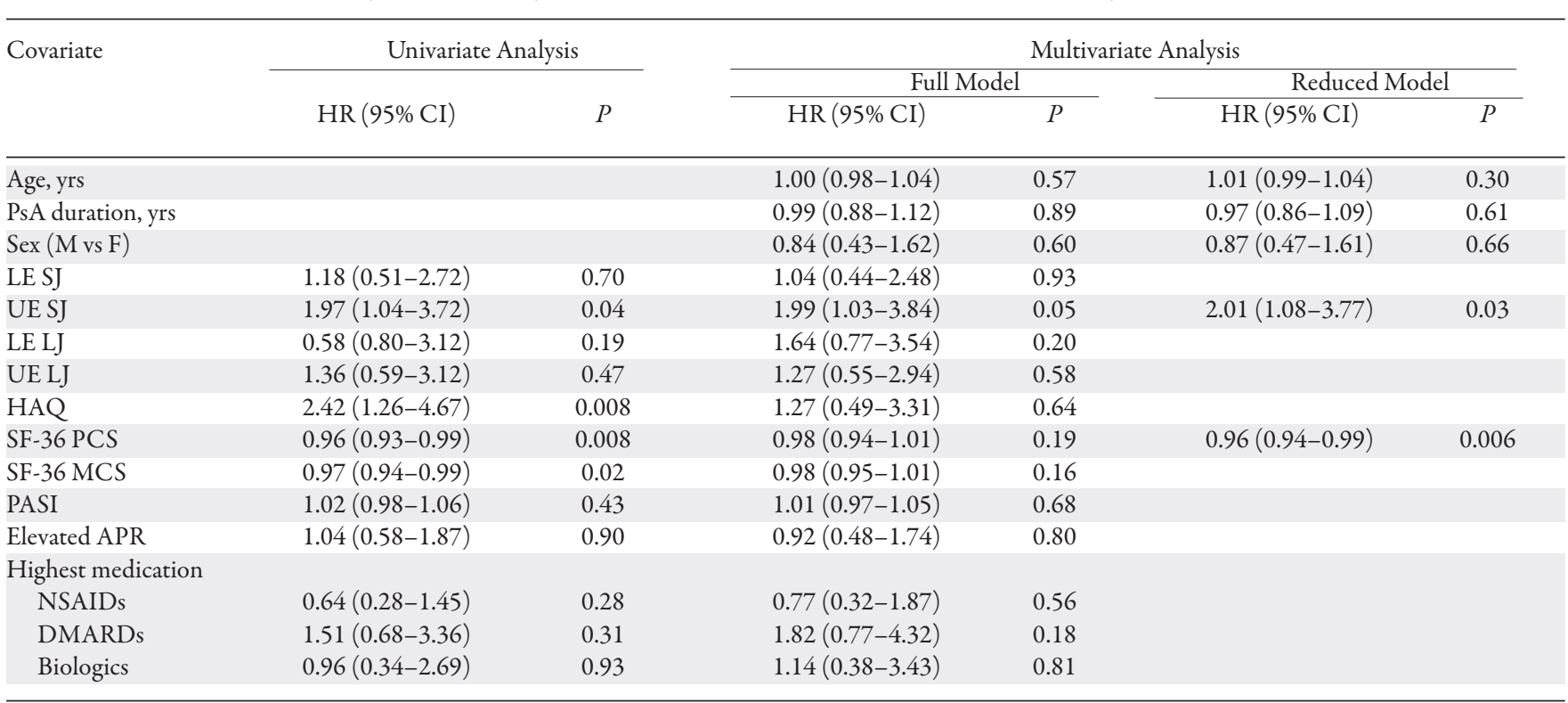

${ }^{a}$ Based on 136 patients with only the covariate information available at each clinic visit (46 progressed to polyarthritis). APR: acute-phase reactant; DMARD: disease-modifying antirheumatic drug; HAQ-DI: Health Assessment Questionnaire-Disability Index; LE: lower extremity; LJ: large joint; MCS: mental component summary score; NSAID: nonsteroidal antiinflammatory drugs; PASI: Psoriasis Area Severity Index; PCS: physical component summary score; PsA: psoriatic arthritis; SF-36: 36-item Short Form Health Survey; SJ: small joint; UE: upper extremity.

\section{DISCUSSION}

Oligoarticular PsA was initially considered the most common pattern of PsA. ${ }^{1}$ Moll and Wright noted that $70 \%$ of their patients presented with oligoarthritis. However, in subsequent studies, the frequency of oligoarthritis has varied widely from $25 \%$ to $65 \%$. $^{2,3}$ 
Table 5. Features associated with progression from oligoarthritis to polyarthritis based on 128 patients (including dactylitis, enthesitis, and axial disease) . $^{2}$

\begin{tabular}{|c|c|c|c|c|c|c|}
\hline \multirow[t]{3}{*}{ Covariate } & \multicolumn{2}{|c|}{ Univariate Analysis } & \multicolumn{4}{|c|}{ Multivariate Analysis } \\
\hline & \multirow[b]{2}{*}{$\operatorname{HR}(95 \% \mathrm{CI})$} & \multirow[b]{2}{*}{$P$} & \multicolumn{2}{|c|}{ Full Model } & \multicolumn{2}{|c|}{ Reduced Model } \\
\hline & & & $\mathrm{HR}(95 \% \mathrm{CI})$ & $P$ & $\mathrm{HR}(95 \% \mathrm{CI})$ & $P$ \\
\hline PsA duration, yrs & & & $0.99(0.86-1.13)$ & 0.86 & $0.96(0.84-1.09)$ & 0.53 \\
\hline Sex (M vs F) & & & $0.90(0.45-1.79)$ & 0.76 & $0.87(0.46-1.62)$ & 0.66 \\
\hline LE SJ & $0.96(0.40-2.34)$ & 0.93 & $0.83(0.32-2.15)$ & 0.69 & & \\
\hline LE LJ & $1.54(0.76-3.13)$ & 0.23 & $1.73(0.77-3.86)$ & 0.18 & & \\
\hline UE LJ & $1.37(0.59-3.16)$ & 0.47 & $1.37(0.58-3.23)$ & 0.48 & & \\
\hline Dactylitis & $1.32(0.50-3.49)$ & 0.58 & $1.81(0.63-5.24)$ & 0.27 & & \\
\hline Enthesitis & $1.68(0.73-3.84)$ & 0.22 & $1.67(0.68-4.07)$ & 0.26 & & \\
\hline Axial disease & $0.57(0.20-1.61)$ & 0.29 & $0.64(0.22-1.88)$ & 0.41 & & \\
\hline PASI & $1.02(0.98-1.05)$ & 0.45 & $1.00(0.97-1.05)$ & 0.67 & & \\
\hline \multicolumn{7}{|c|}{ Highest medication } \\
\hline NSAIDs & $0.60(0.26-1.37)$ & 0.22 & $0.76(0.31-1.86)$ & 0.54 & & \\
\hline DMARDs & $1.13(0.48-2.62)$ & 0.78 & $1.40(0.54-3.62)$ & 0.49 & & \\
\hline Biologics & $0.95(0.33-2.73)$ & 0.92 & $1.29(0.41-4.01)$ & 0.66 & & \\
\hline
\end{tabular}

${ }^{a}$ Based on 128 patients with complete covariate information at each clinic visit (43 progressed to polyarthritis). APR: acute-phase reactant; DMARD: disease-modifying antirheumatic drug; HAQ-DI: Health Assessment Questionnaire-Disability Index; LE: lower extremity; LJ: large joint; MCS: mental component summary score; NSAID: nonsteroidal antiinflammatory drugs; PASI: Psoriasis Area Severity Index; PCS: physical component summary score; PsA: psoriatic arthritis; SF-36: 36-item Short Form Health Survey; SJ: small joint; UE: upper extremity.

We included 407 patients with early disease who entered our clinic within 12 months of diagnosis. Of the 407 patients, 192 (47\%) presented with oligoarthritis based on inflamed joints only. There was no difference in disease duration between those who presented with polyarticular and oligoarticular disease with a mean of $<6$ months of disease duration. Indeed, there were no differences in demographic and clinical features between the 2 groups, aside from the expected difference of the total number of actively inflamed or involved joints as well as the presence of dactylitis and enthesitis. A similar proportion of patients were treated with biologic therapy. Similar observations were noted when the definition of oligoarthritis was based on the number of involved joints, including both inflamed and damaged joints. Of note, the most commonly affected joints even among patients with oligoarticular disease were the small joints of the hands. This is different from the concept that oligoarticular disease tends to involve large joints in the lower extremities.

A study from the Dutch south-west Early Psoriatic Arthritis cohort (DEPAR), an early PsA cohort with an average of 11 months since the onset of symptoms at the time of diagnosis, documented that $56 \%$ of the patients had oligoarthritis at diagnosis. ${ }^{18}$

Clearly, some patients who presented with oligoarthritis progress to develop polyarticular disease. Jones, et al described outcomes in a disease subset of 100 patients with PsA. ${ }^{19}$ Although $63 \%$ of the patients presented with monoarthritis or oligoarthritis, the majority (64\%) progressed to polyarthritis over time. In this study, patients who presented with oligoarticular disease had a lower mean disease duration of PsA (6.6 yrs) compared to those who presented with polyarticular disease (13.9 yrs, $P=0.007)$. The authors concluded that the mode of onset did not predict outcome. In an early PsA study from Dublin, Kane, et a $l^{20}$ reported that $40 \%$ of the patients had oligoarticular PsA, whereas $60 \%$ had polyarticular disease. However, at the follow-up, $39 \%$ of the patients became oligoarticular following treatment. They also concluded that using the pattern of disease to classify patients with PsA may be inappropriate. The frequency of oligoarthritis in this study (39\%) was similar to the frequency of oligoarthritis at presentation in our study $(47 \%)$.

In our study, among 192 inception patients who presented with oligoarthritis, 39\% progressed to polyarticular disease. When patients with dactylitis, enthesitis, and axial disease were not included in the model, upper extremity small joints predicted progression, whereas better physical function protected from progression. However, when the clinical features were added to the model, the only predictor for the evolution to active polyarticular disease was the presence of a lower SF-36 MCS score, suggesting worse mental function. It is not clear why some patients do not progress to polyarthritis. It is possible that treatment may have halted progression to polyarthritis, although the analysis did not reveal that medications played a role. Coates, et al demonstrated that patients with oligoarticular disease who participated in the Tight Control of Psoriatic Arthritis (TICOPA) trial had significant differences between the tight control and standard of therapy groups, although the statistical 
significance was lower than that shown for the whole group of participants. ${ }^{21}$ This study demonstrated that tight control works for oligoarticular as well as polyarticular disease.

Moreover, previous studies using ultrasound showed that oligoarticular patients were reclassified as polyarticular due to subclinical synovitis/disease. ${ }^{22}$ Indeed, in our study, when we included all joints involved-including those that had been damaged-the number of patients with oligoarthritis was reduced.

In conclusion, oligoarticular PsA is similar to polyarticular disease. Lower extremity small joint involvement is associated with polyarticular presentation, but small joint involvement is associated with progression to polyarticular disease.

\section{REFERENCES}

1. Moll JM, Wright V. Psoriatic arthritis. Semin Arth Rheum 1973;3:55-78.

2. Gladman DD, Antoni C, Clegg D, Mease P, Nash P. Psoriatic arthritis - epidemiology and clinical features. Ann Rheum Dis 2005;64 Suppl 2:ii14-7.

3. Dhir V, Aggrawal A. Psoriatic arthritis: a critical review. Clinic Rev Allerg Immunol 2013;33:141-8.

4. Khan M, Schentag C, Gladman D. Clinical and radiological changes during psoriatic arthritis disease progression: working toward classification criteria. J Rheumatol 2003;30:1022-6.

5. Helliwell P, Marchesoni A, Peters M, Barker M, Wright V.A re-evaluation of the osteoarticular manifestations of psoriasis. $\mathrm{Br} \mathrm{J}$ Rheumatol 1991;30:339-45.

6. Ritchlin CT, Kavanaugh A, Gladman DD, Mease PJ, Boehncke WH, de Vlam K, et al; Group for Research and Assessment of Psoriasis and Psoriatic Arthritis (GRAPPA). Treatment recommendations for psoriatic arthritis. Ann Rheum Dis 2009;68:1387-94.

7. Ritchlin CT, Colbert RA, Gladman DD. Psoriatic arthritis. New Engl J Med 2017:376:957-70.

8. FitzGerald O, Ritchlin C. Opportunities and challenges in the treatment of psoriatic arthritis. Best Pract Res Clin Rheumatol 2018;32:440-52.

9. Marchesoni A. Oligoarticular psoriatic arthritis: addressing clinical challenges in an intriguing phenotype. Rheumatol Ther 2018; 5:311-6.
10. Gladman DD, Shuckett R, Russell ML, Thorne JC, Schachter RK Psoriatic arthritis - clinical and laboratory analysis of 220 patients. QJ Med 1987;62:127-41.

11. Gladman DD, Chandran V. Observational cohort studies: lessons learnt from the University of Toronto Psoriatic Arthritis Program. Rheumatology 2011;50:25-31.

12. Gladman DD, Farewell V, Buskila D, Goodman R, Hamilton L, Langevitz P, et al. Reliability of measurements of active and damaged joints in psoriatic arthritis. J Rheumatol 1990;17:62-4.

13. Gladman DD, Cook RJ, Schentag C, Feletar M, Inman RI, Hitchon $\mathrm{C}$, et al. The clinical assessment of patients with psoriatic arthritis: results of a validation study of the SpondyloArthritis Research Consortium of Canada (SPARCC). J Rheumatol 2004;31:1126-31.

14. Stone MA, White LM, Gladman DD, Inman R, Chaya S, Lax M, et al. Significance of clinical evaluation of the metacarpophalangeal joint in relation to synovial/bone pathology in rheumatoid and psoriatic arthritis detected by magnetic resonance imaging. J Rheumatol 2009;36;2751-7.

15. Bond SJ, Farewell VT, Schentag CT, Gladman DD. Predictors for radiological damage in psoriatic arthritis. Results from a single centre. Ann Rheum Dis 2007;66:370-6.

16. Cresswell L, Chandran V, Farewell VT, Gladman DD. Inflammation in an individual joint predicts damage to that joint in psoriatic arthritis. Ann Rheum Dis 2011;70:305-8.

17. Kalbfleisch JD, Prentice RL. The statistical analysis of failure time data. New York: John Wiley \& Sons: 2011.

18. Wervers K, Luime JJ, Tchetverikov I, Gerards AH, Kok MR, Appels CWY, et al. Influence of disease manifestations on health-related quality of life in early psoriatic arthritis. J Rheumatol 2018;45:1526-31.

19. Jones SM, Armas JB, Cohen MG, Lovell CR, Evison G, McHugh NJ. Psoriatic arthritis: outcome of disease subsets and relationship of joint disease to nail and skin disease. $\mathrm{Br} \mathrm{J}$ Rheumatol 1994;33:834-9.

20. Kane D, Stafford L, Bresnihan B, FitzGerald O. A prospective, clinical and radiological study of early psoriatic arthritis: an early synovitis clinic experience. Rheumatology 2003;42:1460-8.

21. Coates LC, Mahmood F, Emery P, Conaghan PG, Helliwell PS. Composite outcome tools in the Tight Control of inflammation in early Psoriatic Arthritis (TICOPA) trial. Ann Rheum Dis 2017;76:1688-92.

22. Wakefield RJ, Green MJ, Marzo-Ortega H, Conaghan PG, Gibbon WW, et al. Should oligoarthritis be reclassified? Ultrasound reveals a high prevalence of subclinical disease. Ann Rheum Dis 2004;63:382-5. 